\title{
ILCEA
}

Revue de l'Institut des langues et cultures

d'Europe, Amérique, Afrique, Asie et Australie

42 | 2021

Le politiquement correct : tabous, normes, transgressions

\section{Au-delà des clivages idéologiques : approches discursives du politiquement correct. - Introduction}

Emmanuelle Prak-Derrington, Dominique Dias et Marie-Laure Durand

\section{OpenEdition}

\section{Journals}

Édition électronique

URL : http://journals.openedition.org/ilcea/11516

DOI : 10.4000/ilcea. 11516

ISSN : 2101-0609

Éditeur

UGA Éditions/Université Grenoble Alpes

Édition imprimée

ISBN : 978-2-37747-251-2

ISSN : $1639-6073$

Référence électronique

Emmanuelle Prak-Derrington, Dominique Dias et Marie-Laure Durand, «Au-delà des clivages

idéologiques : approches discursives du politiquement correct. - Introduction », ILCEA [En ligne], 42 |

2021, mis en ligne le 31 janvier 2021, consulté le 01 février 2021. URL : http://

journals.openedition.org/ilcea/11516; DOI : https://doi.org/10.4000/ilcea.11516

Ce document a été généré automatiquement le 1 février 2021

(c) ILCEA 


\title{
Au-delà des clivages idéologiques : approches discursives $\mathrm{du}$ politiquement correct. - Introduction
}

\author{
Emmanuelle Prak-Derrington, Dominique Dias et Marie-Laure Durand
}

\section{Pour une approche linguistique du politiquement correct}

Le politiquement correct, omniprésent dans les discours publics (politiques, médiatiques, éducatifs, institutionnels, etc.), fait depuis les années 1990 l'objet d'études scientifiques en Allemagne et dans les pays anglo-saxons (voir Reutner \& Schafroth, 2012 ; Suhr \& Johnson, 2016). En France en revanche, il n'a pas encore été théorisé en analyse des textes et des discours, contrairement aux questions voisines que sont la violence verbale et son pendant, la politesse verbale. Le sujet semble ne pas pouvoir être traité indépendamment des convictions politiques, et les études qui lui sont consacrées ne laissent pas de prendre position: pour ou contre le politiquement correct $^{1}$ ? C'est cette bi-polarisation des débats que ce numéro d'ILCEA entend dépasser. Nourri par les travaux du séminaire SéLiA (Séminaire de linguistique allemande) de l'ENS de Lyon, il rassemble les contributions de linguistes, dans les deux sens que prend ce terme en sciences humaines - spécialistes de langues et cultures étrangères, et spécialistes du langage. Il s'agit d'éclairer, au-delà des polémiques, la diversité des mécanismes langagiers que met en œuvre le politiquement correct. Il convient en effet de distinguer deux aspects quand on aborde le politiquement correct en tant que linguiste: la formule elle-même et les vives controverses qu'elle suscite, mais également, au-delà de la formule, l'évolution des normes linguistiques, la question de leur contournement et de leur transgression dans les discours ${ }^{2}$. C'est ce deuxième aspect qui a été privilégié dans ce dossier. 
2 L'expression politiquement correct est calquée sur l'anglais américain political correctness ou politically correct. Utilisée massivement par les mouvements contestataires à partir des années 1960 aux États-Unis, elle a été importée en Europe dans les années 1990 (Appignanesi, 1994) où elle est employée d'abord de manière littérale : politiquement correct «se dit d'un discours, d'un comportement d'où est exclu tout ce qui pourrait desservir socialement un groupe minoritaire» (Nouveau Petit Robert, 2001, s.v. " correct»). Mais l'expression évolue et est progressivement utilisée dans un sens ironique, pour pointer des formes de censure sociale du langage : «Le politiquement correct $[. .$.$] désigne, principalement pour la dénoncer, une attitude qui consiste à$ policer excessivement ou modifier des formulations parce qu'elles pourraient heurter certaines catégories [de la population] (Wikipedia, s.v. "Politiquement correct», consulté le 17 décembre 2020).

3 Est-il possible d'appréhender le politiquement correct de manière scientifique, sans tomber dans l'idéologie? Ce n'est pas un concept linguistique (Kilian, Niehr \& Schiewe, 2010 ; Wierlemann, 2002), mais il porte bel et bien sur des discours et des pratiques langagières. De fait, c'est une formule, au sens de Krieg-Planque (2009)3. Mais ce n'est pas une formule comme les autres, elle se distingue entre toutes par l'étendue de ses domaines d'application - elle peut d'ailleurs se décliner en "écologiquement correct ", " sanitairement correct $t^{4}$ ", « sexuellement correct ${ }^{5}$ », voire " animalement correct $^{6}$ ", etc. Contrairement à ses avatars qui renvoient avant tout à des normes spécifiques de comportement (concernant la santé, l'écologie, la sexualité, etc.), la formule politiquement correct, traduite en français québécois par «rectitude politique» (Hamann, 1998; Boulanger, 2000 : 308), renvoie d'abord à une rectitude langagière. Elle permet, de manière extensive, de qualifier les usages sociaux du langage. «[0]n n'a pas le droit de tout dire, [...] on ne peut pas parler de tout dans n'importe quelle circonstance, [...] n'importe qui, enfin, ne peut pas dire n'importe quoi » (Foucault, 1971:11). On pourrait parler à son propos d'une hyper-formule, d'autant plus plastique et vouée à la circulation qu'elle n'est pas seulement nominale, mais adjectivale - l'adjectif, en tant que qualité, peut être rattaché à un ensemble complètement ouvert de manifestations verbales.

4 L'ancrage socio-historique récent du politiquement correct ne doit pas faire oublier qu'il ressortit à une dialectique discursive immuable, entre tabouisation et euphémisation (Uría Varela, 1997; Reutner, 2009; Reutner \& Schafroth, 2012), que Schafroth décrit comme le "visage de Janus du politiquement correct» (Schafroth, 2010). Par tabouisation, il faut entendre le fait de rendre tabou, de frapper d'interdit une expression, considérée comme inadéquate ou idéologiquement intolérable (« PD » en français, « nigger » en anglais, etc.). L'euphémisation est, elle, le terme générique retenu pour désigner tous les procédés de contournement du tabou verbal (Reutner, 2009). La formule politiquement correct apparaît donc comme l'étiquette contemporaine d'une dialectique inhérente à la production et à la réception des discours, qui met en œuvre un ensemble de phénomènes discursifs a-historiques qui tous ressortissent à la problématique de la désignation (Weinrich, 1970): le recours aux métaphores, aux euphémismes, aux emprunts ("black» pour ne pas dire "noir», "gay " pour ne pas dire «homosexuel »), aux périphrases (" personne en surcharge pondérale » plutôt que " gros »), aux procédés compositionnels (en allemand, « Mitbürger» c'est-à-dire " cocitoyen» pour ne pas dire «migrant»), à la siglaison ( $\mathrm{SDF}$ » pour ne pas dire «clochard»), à l'ellipse, etc. Des manuels qui listent les façons de dire et les prescriptions langagières sont publiés en guise de mode d'emploi ironique du 
politiquement correct $^{7}$, tandis que, inversement, des guides du parler-correct sont recommandés par des institutions ${ }^{8}$.

5 Les comportements langagiers et pratiques discursives du politiquement correct s'inscrivent ainsi dans un mouvement double et antithétique. D'un côté, on trouve celles et ceux qui défendent des formes d'interventionnisme dans la langue : à notre époque, il s'agit de visibiliser et valoriser des groupes perçus comme sous-représentés ou discriminés («personne en situation de handicap » au lieu de «handicapé.e »); de l'autre côté, ceux et celles qui assimilent ces pratiques, au mieux, à une forme de langue de bois bien-pensante ou de "néoconformisme", au pire, à une tentative de manipulation et de désinformation (Volkoff, 2001:50) (voir «les dommages collatéraux » pour désigner les victimes civiles dans le langage militaire). En France, l'actuelle polémique autour d'un langage inclusif fournit sans doute l'exemple le plus éclatant de ce caractère bifrons. L'idéologie du politiquement correct promeut une discrimination positive et un égalitarisme sociétal, qui viennent alors s'inscrire en faux contre le purisme de la langue (sur la langue et le purisme, voir Paveau \& Rosier, 2008).

C'est cette articulation complexe entre langue et discours, politique, idéologie et société qui rend le politiquement correct si difficile à théoriser (Forster, 2010). Tabous et normes sont sans cesse en mutation et leur reconfiguration permanente participe au caractère insaisissable du politiquement correct, qui révèle alors le système de valeurs, autant que la place idéologique accordée à la langue, dans un contexte et à un moment donnés de l'histoire.

7 Certaines périodes, plus que d'autres, cristallisent les passions sur la langue et favorisent la tabouisation (entre autres le courant de la préciosité à l'âge baroque). L'originalité des débats sur le politiquement correct ne réside donc pas dans la dialectique de la désignation (il y a toujours eu des mécanismes de censure et d'autocensure), mais dans la manière radicale dont ses termes sont posés. Des interdits qui pouvaient n'être qu'intériorisés peuvent au $\mathrm{XxI}^{\mathrm{e}}$ siècle être passibles de sanction, des mots déclarés incorrects sont brandis de manière décontextualisée pour condamner ceux qui les ont proférés. Une universitaire d'Ottawa ${ }^{9}$ a pu être suspendue pour avoir prononcé le $N$-word, dans un cours pourtant consacré à la question de la réappropriation de ce terme par la communauté afro-américaine - ce qu'en sociologie, on appelle le « retournement du stigmate » (Goffman, 1975).

On pourrait multiplier à l'envi les exemples de ce genre de dérives surévaluant le poids de mots isolés réputés "incorrects", au détriment de leur emploi en discours. Ce numéro d'ILCEA fait le choix, au contraire, de ne pas se limiter à une approche prioritairement lexicale du politiquement correct. En ces temps de radicalisation des opinions, il nous semble essentiel de mettre au jour la complexité linguistique des phénomènes de désignation, qui excède tout mécanisme de censure.

\section{Présentation des articles}

Dans l'article liminaire, Patricia von Münchow propose de recourir à l'appareil théorique de l'analyse du discours pour comprendre la prescription et l'interdiction verbale au sein d'une même culture discursive. À l'aide d'une grille d'analyse permettant, à partir de procédés discursifs plus ou moins saillants, d'identifier des normes et des représentations en vigueur, sa méthode permet d'éviter l'écueil d'une approche trop lexicale du politiquement correct pour lui préférer un ancrage discursif. 
Elle illustre le fonctionnement de cette grille d'analyse à partir d'un corpus de manuels scolaires d'histoire français et allemands traitant du nazisme et de la Seconde Guerre mondiale. Ces deux questions revêtant un véritable enjeu mémoriel dans un contexte didactique, elles sont particulièrement éclairantes sur la perception des tabous et des évolutions normatives.

10 L'existence de tabous langagiers dans une société implique également la transgression de ces tabous et donc la question du politiquement incorrect. Maria Saltykov s'interroge ainsi sur la création et l'évolution de la formule " politiquement incorrect » dans les discours savant, médiatique et numérique francophones et anglophones. En s'appuyant sur les méthodes de l'analyse qualitative du discours et de l'argumentation, elle montre comment le politiquement incorrect se fonde en réaction au politiquement correct, avant de proposer une définition discursive du politiquement incorrect, et de mettre en évidence les enjeux argumentatifs qu'il revêt. Le politiquement incorrect, qui peut se concevoir comme une réaction à des normes de communication restrictives, peut être aussi bien perçu comme une libération dangereuse de la parole ou encore comme l'expression d'une nécessaire impertinence. La difficulté à le saisir et à le catégoriser réside dans le fait que des discours transgressifs peuvent devenir à leur tour des discours dominants.

11 L'omniprésence du politiquement correct est également liée au fait qu'il prend forme dans des phénomènes sociétaux médiatisés et souvent controversés dont la deuxième section entend donner un aperçu. Le phénomène par excellence qui établit un lien direct entre langage symbolique et représentation est celui du langage inclusif, animé par la volonté de rendre visibles les femmes à travers le marquage du féminin dans des langues où le masculin a valeur de générique. Dans sa contribution, Nathalie Schnitzer dresse un état des lieux des pratiques du langage inclusif en France et en Allemagne. Elle s'interroge sur leur avenir et sur leur capacité à produire plus d'équité. Elle revient dans un premier temps sur les objections formulées contre le langage inclusif et sur les contre-arguments que l'on peut leur opposer, avant de proposer une étude contrastive français-allemand des faits de langue concernés et des stratégies de marquage mises en œuvre dans les deux langues.

12 Un autre phénomène qui partage cette même croyance dans les vertus du langage est celui de la Leichte Sprache (LS), qu'Odile Schneider-Mizony nous présente dans sa contribution. Il s'agit de la langue facile à comprendre utilisée pour "traduire » de nombreux documents à destination des personnes rencontrant des déficits cognitifs. L'exemple de cette forme d'interventionnisme langagier à visée morale illustre les ambitions nourries par toute entreprise politiquement correcte: agir sur la langue pour agir sur la société et la rendre plus juste. Après avoir rappelé la genèse de la LS, et illustré certaines de ses applications, l'auteure montre que les règles édictées pour rendre la langue accessible à toutes et à tous sont d'une efficacité plus que discutable.

13 Les procédés d'atténuation des réalités peuvent également être motivés par une volonté délibérée de masquer, voire de tromper. Les choix de désignation revêtent en tout cas des enjeux de perception des réalités. Telle est la question que pose Caroline Benedetto dans sa contribution portant sur l'évolution diachronique des termes et expressions de l'économie collaborative en anglais. Elle montre que l'émergence de nouvelles désignations répond certes à des aspirations actuelles des citoyens au partage et à la solidarité. Il n'en reste pas moins que ces termes et expressions désignent des pratiques économiques qui ne reposent pas que sur l'entraide, tout en véhiculant une 
certaine vision économique et sociale du monde. On comprend mieux dans quelle mesure la désignation est susceptible de contribuer à façonner les représentations du réel socio-économique.

La dernière section de ce volume est consacrée à la question délicate de la désignation des personnes susceptibles d'être discriminées. Même si l'on s'affranchit de l'encombrante étiquette du politiquement correct, il n'en reste pas moins que nos pratiques langagières nous invitent à faire coïncider la chose avec le mot juste et nous conduisent dans certains cas à un dilemme : comment désigner l'Autre? Le principe affiché du politiquement correct est de supprimer des dénominations considérées comme stigmatisantes pour en adopter de plus respectueuses. Partant de ce constat, Sylvain Farge s'intéresse à la dénomination des personnes en surpoids dans un corpus de presse germanophone pour déterminer si une axiologie peut être associée à une dénomination en discours. Cette étude à l'interface entre langue et discours s'appuie sur les outils de la statistique lexicale et notamment de l'analyse de correspondances multiples (ACM). S'il apparaît évident qu'un lexème peut véhiculer un ensemble de représentations et de connaissances, il n'est pas certain que sa simple suppression suffise à faire disparaitre ces représentations. Par ailleurs, les stratégies d'euphémisation du surpoids ne sont pas sans poser des problèmes morphologiques et référentiels : certaines constructions lexicales vont à l'encontre du principe d'économie de la langue et l'euphémisation induit une certaine imprécision référentielle et sémantique.

Il en va de même pour la désignation de l'étranger qui prend un caractère politique et argumentatif dans le contexte des crises migratoires. Tel est le sujet exploré par Philippe Verronneau dans une contribution consacrée aux problèmes de désignation dans les prises de parole de la chancelière allemande lors de la crise migratoire de 2015. La désignation de l'étranger illustre parfaitement le principe des chaines euphémistiques : les désignations évoluent en fonction des périodes, supprimant des expressions jugées dévalorisantes au profit de termes plus neutres, mais qui ne tardent pas à se charger à leur tour d'une connotation péjorative. L'enjeu pour les responsables politiques dans un contexte aussi délicat est de ne pas réduire les populations concernées à leur condition migratoire tout en cherchant à légitimer une politique migratoire parfois controversée.

Dans un contexte cette fois didactique, Laure Gautherot analyse pour sa part la désignation des groupes migratoires étrangers dans les manuels d'enseignement de l'allemand en France. Le large corpus de manuels qu'elle explore lui permet de mettre au jour les moyens lexicaux mis en œuvre pour désigner l'étranger entre 2001 et 2019. Cet empan assez large qui couvre deux décennies montre comment les concepteurs de manuels s'efforcent d'intégrer les évolutions sociolinguistiques de l'allemand contemporain. Les évolutions observées traduisent parailleurs des stratégies discursives antidiscriminatoires qui présentent des enjeux pédagogiques. Ces évolutions reflètent en outre les changements sémantiques et idéologiques en vigueur à une période donnée.

17 Enfin, on a pu constater que les formules politiquement correct et politiquement incorrect ont un ancrage socio-historique bien précis et que les tabous langagiers et leur traitement évoluent en fonction des époques. Pedro Duarte propose de mettre en perspective ces deux notions antinomiques en s'interrogeant sur leur pertinence dans l'Antiquité. Avec toutes les précautions d'usage qui prévalent à l'étude de concepts 
modernes appliqués à une langue et une civilisation antiques, il examine quelques dénominations de la plèbe dans la Rome républicaine et les métadiscours autour de ces dénominations. L'aspiration à une modération des propos pour ne pas blesser l'autre et à celle d'un franc-parler ne sont pas étrangères à l'Antiquité et s'incarnent même dans des courants philosophiques influents. Les questions de dénomination autour d'un groupe existent bel et bien et elles accompagnent en partie des revendications d'ordre sociopolitique, mais elles ne créent pas pour autant les conditions d'une opposition virulente entre les défenseurs et les opposants d'un interventionnisme langagier.

Loin d'avoir épuisé la question du politiquement correct, ce premier numéro de revue consacré à cette problématique en donne néanmoins un aperçu documenté par des corpus français, anglais et allemands, dans des contextes différents, qui montrent à la fois l'omniprésence de ce questionnement et l'urgence qu'il y a à comprendre les mécanismes qui sous-tendent les phénomènes d'interdits langagiers et de prescriptions normatives.

\section{BIBLIOGRAPHIE}

APPignANESI Lisa (1994), «Liberté, Égalité, Fraternité: PC and the French », S. Dunant (dir.), The War of Words: The Political Correctness Debate, Londres : Virago.

BocK-CôTÉ Mathieu (2019), L'empire du politiquement correct : essai sur la respectabilité politicomédiatique, Paris : Les Éditions du Cerf.

BOULANGER Jean-Claude (1986), Aspects de l'interdiction dans la lexicographie française contemporaine, Tübingen : Niemeyer.

FASSIN Éric (2003), « Penser la discrimination positive », D. Borrillo (dir.), Lutter contre les discriminations, Paris : La Découverte, 55-68.

FASsIN Éric (2019), « Le politiquement correct et les médias », Mediapart (blog), en ligne sur <https://blogs.mediapart.fr/eric-fassin/blog/230119/le-politiquement-correct-et-les-medias> (18 décembre 2020).

FORSTER Iris (2010), « Political Correctness / Politische Korrektheit », Bundeszentrale für politische Bildung, en ligne sur bpb.de: <www.bpb.de/politik/grundfragen/sprache-und-politik/42730/ politische-korrektheit> (18 décembre 2020).

FoucAult Michel (1971), L'ordre du discours, Paris : Gallimard.

GoffMAN Erving (1975), Stigmate : les usages sociaux des handicaps, traduit par Alain Kihm, Paris :

Minuit.

HAMANN Jean (1998), « Politiquement incorrect ? », Le Fil, Le journal de la communauté universitaire, en ligne sur <https://archives.nouvelles.ulaval.ca/Au.fil.des.evenements/1998/06.11/ politique.html> (18 décembre 2020).

KILIAN Jörg, NIEHR Thomas \& SCHIEWE Jürgen (2010), Sprachkritik. Ansätze und Methoden der kritischen Sprachberatung, Berlin, New York : De Gruyter. 
KRIEG-PlANQUE Alice (2009), La notion de « formule » en analyse du discours : cadre théorique et méthodologique, Besançon : Presses universitaires de Franche-Comté.

PAVEAU Marie-Anne \& Rosier Laurence (2008), La Langue française : passions et polémiques, Paris : Vuibert.

REUTNER Ursula \& SCHAFRoth Elmar (dir.) (2012), Political Correctness. Aspects politiques, sociaux, littéraires et médiatiques de la censure linguistique, Francfort-sur-le-Main : Peter Lang.

REUTNER Ursula (2009), Sprache und Tabu. Interpretationen zu französischen und italienischen Euphemismen, Tübingen : Niemeyer.

SCHAFROTH Elmar (2010), « Die Janusköpfigkeit der Political Correctness. Dargestellt am Beispiel der sprachlichen Feminisierung in Quebec und Frankreich und an anderen Phänomenen », Zeitschrift für Kanada-Studien, 30(2), 101-124.

SuHR Stephanie \& JoHnson Sally (2003), « Re-Visiting 'PC': Introduction to Special Issue on 'Political Correctness' », Discourse \& Society, 14(1), 5-16.

URÍA VARELA Javier (1997), Tabú y eufemismo en latín, Amsterdam : A. M. Hakkert.

VolKoff Vladimir (2001), Manuel du politiquement correct, Monaco : Éditions du Rocher.

WEINRICH Harald (1970), Linguistik der Lüge. Kann Sprache die Gedanken verbergen?, édité par Deutsche Akademie für Sprache und Dichtung, Heidelberg : L. Schneider.

WierLemann Sabine (2002), Political Correctness in den USA und in Deutschland, Berlin : E. Schmidt.

\section{NOTES}

1. Voir en sociologie l'opposition entre les chercheurs qui revendiquent un ancrage à gauche et qui voient dans le politiquement correct le moyen de lutter contre les discriminations (par exemple Fassin, 2003, 2019, etc.) et ceux qui sont ouvertement conservateurs et condamnent le politiquement correct sans ambages (voir l'ouvrage de Bock-Côté, 2019).

2. Sur cette distinction, voir l'introduction de Suhr et Johnson (2003).

3. Krieg-Planque définit la formule comme « un ensemble de formulations qui, du fait de leurs emplois à un moment donné et dans un espace public donné, cristallisent des enjeux politiques et sociaux que ces expressions contribuent dans le même temps à construire » (2009:7).

4. Employé entre autres par André Comte-Sponville: voir <www.lepoint.fr/economie/andrecomte-sponville-ne-tombons-pas-dans-le-sanitairement-correct[...].php\#>.

5. Voir l'album de Wolinski paru en 1996.

6. Mot d'ordre de fin d'année 2007 de la Fondation 30 millions d'amis: voir < www. 30millionsdamis.fr/actualites/article/206-animalement-correct/>.

7. Par exemple, Frédéric Mathieu, Jamais sans ma Novlangue! Le décodeur de poche, Paris : The Book Edition, 2014.

8. C'est le cas du Manuel d'écriture inclusive, promu par le ministère chargé de l'Égalité entre les femmes et les hommes, de la Diversité et de l'Égalité des chances, et qui est téléchargeable gratuitement via le lien : <www.motscles.net/ecriture-inclusive>.

9. «Ottawa professor apologizes for using N-word, regrets growing controversy», $C B C$, 21 octobre 2020, <www.cbc.ca/news/canada/ottawa/professor-apologizes-university-ofottawa-1.5770946>. 


\section{AUTEURS}

\section{EMMANUELLE PRAK-DERRINGTON}

ENS de Lyon, ICAR.

Emmanuelle Prak-Derrington est maîtresse de conférences HDR en linguistique allemande à l'ENS de Lyon et membre du laboratoire ICAR (page personnelle). Ses travaux développent une linguistique anthropologique, en privilégiant l'étude des catégories à la fois ontologiques et linguistiques (la personne, le temps, la répétition). Elle vient de publier un ouvrage Magies de la répétition.

\section{DOMINIQUE DIAS}

Université Grenoble Alpes, ILCEA4.

Dominique Dias est maître de conférences en linguistique allemande à l'Université Grenoble Alpes. Il est membre du laboratoire ILCEA4 et travaille au sein de l'équipe du Gremuts en linguistique textuelle sur les genres de discours. Ses dernières publications portent notamment sur la définition et l'évolution des pratiques métatextuelles comme le fact-checking ou les recensions journalistiques.

\section{MARIE-LAURE DURAND}

Université Paul Valéry - Montpellier 3, CREG.

Marie-Laure Durand est maître de conférences en linguistique allemande à l'Université Paul Valéry - Montpellier 3 et membre du CREG (Centre de recherches et d'études germaniques). Ses recherches portent sur les constructions détachées, dans une approche morphosyntaxique et pragmatique, et sur la structuration du discours et de l'argumentation. 